\title{
Spatial Design, Designers and Users: Exploring the Meaning of Multi-party Service Cognition
}

\author{
Tom Hope ${ }^{1}$, Mizuki Oka ${ }^{2}$, Yasuhiro Hashimoto ${ }^{2}$, and Myeong-Hee Lee ${ }^{3}$ \\ ${ }^{1}$ Tokyo Institute of Technology, 2-12-1-E3-3 \\ Ookayama, Meguro-ku, Tokyo 152-8550, Japan \\ ${ }^{2}$ University of Tokyo, Bunkyo-ku, Tokyo, Japan \\ ${ }^{3}$ Design Team Matt, Tokyo, Japan \\ tomhope@ipo.titech.ac.jp, lee@mattoct.jp, \\ mizuki@cks.u-tokyo.ac.jp, \\ hy@sys.t.u-tokyo.ac.jp
}

\begin{abstract}
This paper examines service cognition from the perspective of the design of public or semi-public spaces. Results are presented from a workshop with students using an approach and system we developed to engage users with the design of their communal space. The paper ends with a brief discussion of these results, which suggest that multi-party service cognition has features that must be addressed in the design of systems, particularly in the context of spatial design.
\end{abstract}

Keywords: service cognition, design, architecture, workshops, universities.

\section{Introduction}

Many changes happened in engineering and industry during the end of the $20^{\text {th }}$ century, with the greatest of these continuing globally to the present day. Broadly speaking, industrialized nations moved from heavy manufacturing as a base to knowledge economies [1], coinciding with, and supported by, ever-increasing computing power. While developing economies took up the role of being producers of basic goods in their main industries, the 'post-industrial' economies focused on developing their service sectors [2]. With the rise of 'manufactured risk' [3] comes an urgent need to understand and improve the management and control of highly sophisticated technical systems [4]. In concert with this area of research, studies of the cognition of those involved in service in other areas, and of cognition in organizations more generally have continued. "Service cognition" as a research field is potentially very broad and can therefore denote the cognition of individuals providing services in many varied fields. In this paper we emphasize the other part of service systems-the users of services - and particularly focus on the interrelated nature of providers and consumers/users. Additionally, while research has been done on various systems and settings, there is still a need to more fully understand service cognition in the realm of architecture and design. This paper consequently aims to partly fulfill this need. The paper is organized as follows. In the rest of this section we briefly explore the 
relationship between service cognition and spatial design. In section 2 we introduce a system we have developed to aid the participatory design of semi-public spaces. In section 3 we present some results and conclude with a discussion in section 4 .

\subsection{Spatial Design as Service}

Although not a new development in theory, in practice the commercial desire to create spaces that can be used for multiple purposes, or "flexible spaces", has developed into a prominent aspect of architecture only in recent years [5]. This can take the form of movable walls and furniture, which allow the use of the space to be changed according to different needs of clients, and may even enable changes to be made within the course of a week or day. Architects are usually involved only in the initial construction of buildings, leaving decisions about use to be done by owners. Hence, with the ability to transform spaces after their construction, if buildings are to be used by people other than themselves, the managers and administrators of those spaces take on an additional role of designers-or, rather, 're-designers'. It is in this sense that the management of a space and reworking of layout is clearly a service. If the space is public or semi-public, such as a gallery, university campus or office, the providers and managers of the space are part of a complex system of provision and use.

\subsection{Cognition, Service and Participatory Design}

We can note that while traditionally architects and designers of spaces may be separated from the users - the architects and interior designers working prior to users being in the space-administrators of buildings necessarily need to engage with users at some level. However, their evaluations of spatial use might typically take the form of surveys [6]. While this process may be iterative, it does not involve the users directly with the re-designing of the space and may struggle to fully manage to comprehend the multiple layers of spatial use [7]. A participatory design [8] approach may be a way to more fully involved users in the provision of appropriate spatial design. We assert that involving the users in the (re-)designing of the spaces they use will be a useful way to create functional, flexible spaces that 'fit' with changing needs. However, the potential mis-match of cognition between professionals and nonprofessionals and between users and other users could create difficulties. The appropriate inclusion of digital technologies in this process may benefit the administrators, users, and professional designers.

Studies that use mobile or pervasive technologies to understand spatial use are multiple [9, 10]. Unfortunately, while the data produced is useful and may be generalised, it is difficult to gain access to the actual beliefs or desires of users at the time of use. It should also be recognised that people involved in the service systemdesigners, administrators and users-will be unlikely to have matching cognition. Indeed, the differences in the beliefs or assumptions about use of original architects and eventual users may be fundamental to why spaces are unused or under-used. In a multi-party process of redesigning space, a system is needed that allows all participants to work together and to somehow 'see' each others' cognition. 
In the rest of this paper we therefore do not intend to present a model of service cognition in spatial design, but instead hope to illuminate one way of determining this cognition. We demonstrate a system where members-users or designers of spacecan work together in a participatory framework to create new ways of use.

\section{The Pingpong Project and Platform}

Participants involved in services, whether on the receiving end-in this case the users of a space-or the other side, as designers, architects or administrators, necessarily plan and evaluate at different levels. It has been shown that visualisation, whether in the form of sketches [11] or more elaborate diagrams, is a significant part of design [12]. This visualisation may be considered to be a way to 'externalise' cognition, a practical cognitive map which can be utilised by others in the design process. Our project, known as 'pingpong' ${ }^{1}$ aims to structure design processes using multidisciplinary research related to computer science, and technologies such as webmining, natural language processing and semantic web. We have developed a platform and set of tools to facilitate this aim, and have to date explored its use in several design workshops held at different university campuses.

\subsection{Pingmap Application}

For the users, the system consists of a mobile application installed on mobile phones and a web-based browser visualization. The platform itself is the application, a human behavior extraction engine and a visualization map. Upon launching the application, users see a start screen and then a screen with a map of the university campus (or space that will be studied), seen in Fig.1 ( $a$ and $b$ ).

The user touches the screen to locate themselves on the map, after which an input box is displayed where they insert some text (Fig. 1c). The text is then uploaded to the Web using Twitter, the micro-blogging service ${ }^{2}$. Because we used Twitter as an

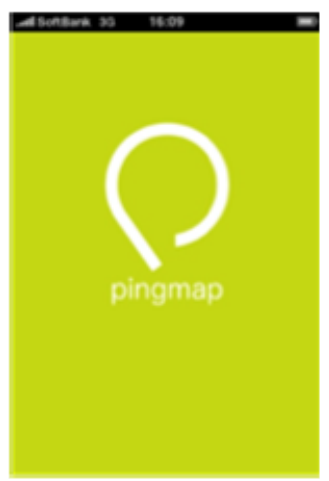

(a) start screen

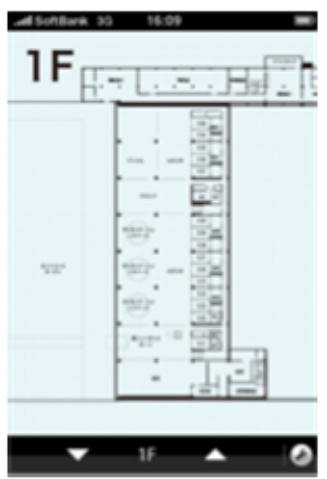

(b) campus map

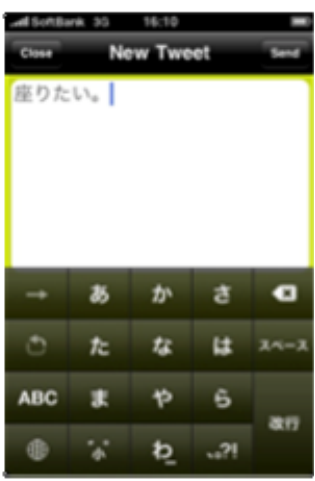

(c) text input

Fig. 1. The Pingmap application

\footnotetext{
${ }^{1}$ http://www.pingpong.ne.jp/

${ }^{2} \mathrm{http} / / /$ twitter.com
} 
underpinning infrastructure to collect the data, the number of characters is limited to 140. This data is collected along with user's account name, posted text, a hash-tag, Xaxis of the map, Y-axis of the map, and the floor number of the space. As an example, if a user with an account name of miz oka posts a text "Coffee now", the location information of the map (X-axis, Y-axis, and the floor number) along with the hash-tag specified for the workshop are automatically attached and will produce the following post:

Miz_oka, coffee now, \#ppfun, 283, 142, 5. 2010-02-15 10:35:33 JST

\subsection{Pingpong Engine}

The pingpong engine, a human behavior extraction engine, analyses each post from the pingmap application and extracts human actions. For analysis, we define 'human behavior' in terms of three linguistic elements (subject, verb and object). Each user's posted text (referred to as tweet from herein) is collected using the API and natural language processing techniques are applied to extract these three elements as one human action. Specifically for example, from a tweet "I read a book", the engine extracts "I", "read", "a book". Further details of the natural language processing applied and specifically the methods of dealing with ungrammatical and fragmented text in tweets can be found in [13].

\subsection{Pingpong Map}

The pingpong map visualizes the collected data in a web-based browser. The visualization is provided in the form of a map in which the verb is extracted from each post and plotted using its location information. This visualization is intended to enable collaboration between users, provided so as to facilitate the sharing of information among workshop participants. Fig. 2 shows a snapshot of the pingpong Map developed for a workshop described below. The left part of the screen shows the tweets in a timeline, but the right side is a map of the space showing only the verbs plotted in the location where the tweets were posted. The reason for showing only verbs is to allow the user to grasp the general content of the tweet and location without needing to read the entire text. Verbs rather than nouns allow the grasping of content in sentences [14]. Clicking on a verb on the map prompts the appearance of its original tweet in the timeline, and clicking on the timeline tweets highlight them in the map for easy reference.

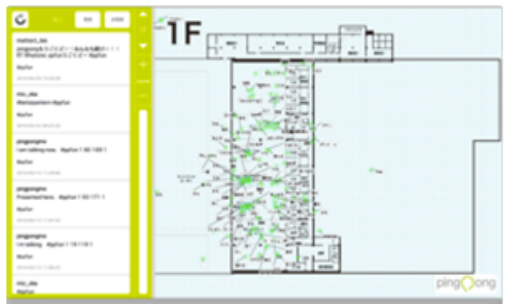

(a) visualization map snapshot

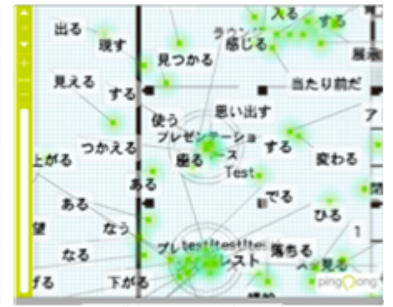

(b) zoomed-in

Fig. 2. Screenshot of the Pingpong Map 


\subsection{Workshop Study}

To evaluate the tools in a real-world system, a series of workshops were conducted in university campuses. Here we report on one, held to examine the use of a campus of a university in the north of Japan.

The workshop was conducted with a total of 15 people consisting of 12 undergraduate and graduate students from the university under study and three students from other universities. Students were recruited via an introductory lecture we gave at the university, the mailing lists of the staff and the students, and advertising it on our project website as well as the website of the public relations department of the university. Most of the participants majored in either computer science or information science. The participants were divided into three groups with each group consisting of five students. We also made the set of tools developed for the workshop available for the people who have internal access to the university local network to access, allowing anyone to download the software. This was advertised a month in advance and posted on our blogs as well as the university's website.

The workshop consisted of six phases, namely observation, analysis, idea generation, prototyping, implementation and use. These phases were held on different days, depicted in Fig. 3.

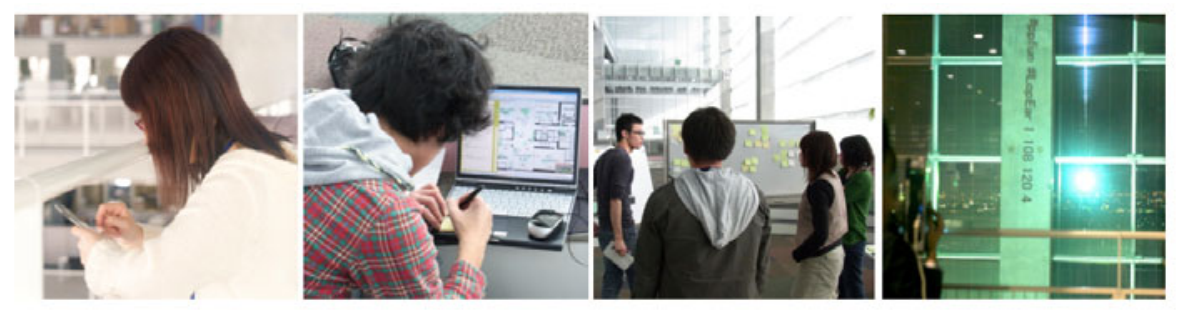

Fig. 3. Day 1: data collection, Day 2: analysis, Day 3: idea generation, Day 4 implementation

On the first day, students used the pingpong application on mobile phones walking around the building. They were told to post tweets freely. This data was collected by the system and used on the second day where workshop participants were provided with the pingpong map visualization tool. Using the tool, the students attempted to understand the current and desired use of the campus space. On the following two days, ideas about new ways of using the space were generated based on the earlier analysis, and finally prototyped to see if they were workable. The workshop participants presented their ideas and prototypes to each other on this last day. Throughout the workshop, hand-held video was captured of student interactions with the system, along with separate audio data during the second and third days of group discussion in order to give observational data for analysis.

\section{Results}

The workshop proved to be an effective way to see use of the system in a real-world setting. Firstly, the video of the students while collecting data (tweeting) showed that 
data on spatial usage could be collected in a short time with minimum instruction. It was initially noticeable that the tweets produced by students around the space could apparently be categorized into whether they were about a static observation about the physical space, or whether students-most of whom were regular users of the campus - noticed some change in the space since a previous visit (described more fully in [14]. This is significant as it is information that could not be gained easily by an external designer, but that could prompt users to think of a possible way to redesign the space.

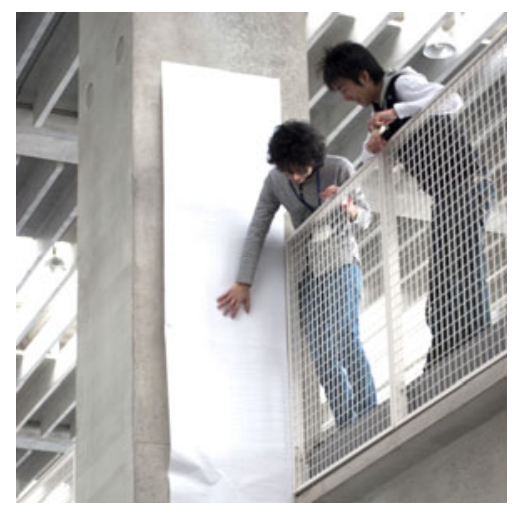

Fig. 4. Students preparing their implementation

The second day was initially frustrating for some of the students, as the pingpong map did not function according to how they desired it to. Initial interest was on the data that they had produced themselves and which would have been the topic of their discussion and design. But the way the tweets had been processed by the system made it very difficult to simply find one's own posts without seeing those of other people. In effect the system pushed workshop participants toward comprehending other users' experience and desire for the space. The freedom that students had been given about their posting of tweets with the system also highlighted the necessity to recognize the different levels of spatiality, namely that in addition to physical environmental observations, social interactions between students led to posting - much as we may expect in non-workshop 'real' settings. This can be seen in Extract 1, which shows two tweets, with the latter one prompted by the sight of the first.

\section{[Extract: Social Interaction Tweets] \\ Student A}

Waving at a person who got off from the elevator. 2010-02-16 13:42:45 JST

Student B

This may be useful for finding a person. 2010-02-16 13:43:27 JST

The beliefs held about the usage of the space were therefore shown to emerge from interactions with others in the same building. While it would not be impossible for individual administrators and designers to gain access to this data, pingong and the workshop format facilitated this easily. 
Students used the visualization to generate ideas about redesigning the space to more effectively represent desired use and all were able to decide an implementation to prototype on the final day. These included ways to improve navigation information displayed in the building and to more fully utilize unused areas.

\section{Discussion}

This study briefly introduced a system to aid in the redesigning of semi-public spaces. Though the study at this stage did not involve professional designers, the workshop participants found the system useful in their development of a new spatial designs. It is envisaged that the logical extension of a system such as pingpong would be the ability to collect data naturalistically, such as that produced in users' daily lives through micro-blogging or other mobile-online behavior. This will be a next stage of our future work. Nevertheless, the workshop itself provided a significant benefit that data could be collected in a short time and the producers of that data were then able to join and collaborate around a representation of their use and desire for use of the space. As such, a workshop format with a system like pingpong may be beneficial to administrators of buildings in the short term at least.

The study illustrates that service cognition in the context of participatory design is complicated by the involvement of multiple parties, each with differing understandings of present and desired use. While the workshop this time included one type of user - the students - the system prompted their recognition of beliefs and desires in the other groups. This would only be emphasized more with the addition of professional architects and administrators. Although the pingpong system has been developed from an emphasis in participatory spatial design, we believe that it may have use in other types of multi-party service, as it can enable, even strongly prompt, participants to visualize and discuss their cognitive maps.

\section{References}

1. OECD: The Knowledge-Based Economy, OECD, Paris (1996)

2. McMichael, P.D.: Development and Social Change: A Global Perspective, 4th edn. Sage/Pine Forge, Thousand Oaks, CA (2008)

3. Giddens, A.: Runaway World: How Globalization is Reshaping Our Lives, Profile, London (1999)

4. Kanno, T., Nakata, K., Furuta, K.: A method for conflict detection based on team intention inference. Interact. Comput. 18(4), 747-769 (2006)

5. Kronenburg, R.: Flexible: Architecture that Responds to Change. Laurence King Publishers (2007)

6. CABE: Creating Excellent Buildings: A Guide for Clients. (2011), http: / / www. cabe.org.uk/publications / creating-excellentbuildings

7. Paay, J., Kjeldskov, J., Howard, S., Dave, B.: Out on the Town: A Socio-Physical Approach to the Design of a Context-Aware Urban Guide. ACM Trans. Comput.-Hum. Interact. 16(2), Article 7 (June 2009) 
8. Blomberg, J., Giacomi, J., Mosher, A., Swenton-Wall, P.: Ethnographic field methods and their relation to design. In: Schuler, D., Namioka, A. (eds.) Participatory Design: Principles and Practices, Hillsdale (1993)

9. Randell, C., Muller, H.L.: Low cost indoor positioning system. In: Abowd, G.D., Brumitt, B., Shafer, S. (eds.) UbiComp 2001. LNCS, vol. 2201, p. 42. Springer, Heidelberg (2001)

10. Lee, S.-W., Mase, K.: Activity and Location Recognition Using Wearable Sensors. IEEE Pervasive Computing 1(3), 24-32 (2002)

11. Gero, J.S., Tversky, B., Purcell, T. (eds.): Visual and Spatial Reasoning in Design II, Key Centre of Design Computing and Cognition, pp. 271-282. University of Sydney, Australia (2001)

12. Won, P.H.: The comparison between visual thinking using computer and conventional media in the concept generation stages of design. Automation in Construction 10(3), 319-325 (2001)

13. Oka, M., Myeong-Hee, L., Hashimoto, Y.: Pingpong: Supporting Collaborative Design Work by Mining Human Behaviors in the Field. In: The Second International Service Innovation Design Conference (2010)

14. Langacker, R.: The Foundations of Cognitive Grammar: Theoretical Prerequisites, vol. 1. Stanford University Press, Stanford (1987) 